\title{
Validity of the French version of the Core Outcome Measures Index for low back pain patients: a prospective cohort study
}

\author{
Stéphane Genevay • Marc Marty • Delphine S. Courvoisier • Violaine Foltz $\cdot$ Geneviève Mahieu • \\ Christophe Demoulin • Agnieszka Gierasimowicz Fontana $\cdot$ Michael Norberg • Pierre de Goumoëns • \\ Christine Cedraschi · Sylvie Rozenberg • Section Rachisde la Société Française de Rhumatologie
}

Received: 8 October 2013/Revised: 17 April 2014/Accepted: 18 April 2014/Published online: 15 July 2014

(c) Springer-Verlag Berlin Heidelberg 2014

\begin{abstract}
Purpose Among the many questionnaires available to evaluate low back pain (LBP) patients, the Core Outcome Measures Index (COMI) has the unique advantage to investigate five dimensions using seven short questions. The aim of this study was to explore additional properties of the questionnaire in a French-speaking non-surgical population.

Methods This study was conducted on 168 patients suffering from subacute or chronic LBP and followed up for 6 months in three French-speaking countries. In addition to basic psychometric properties (e.g., construct validity, floor and ceiling effect, reproducibility), internal validity was analyzed by a factor analysis using Cronbach's alpha.
\end{abstract}

Electronic supplementary material The online version of this article (doi:10.1007/s00586-014-3325-8) contains supplementary material, which is available to authorized users.

S. Genevay $(\bowtie)$

Department of Rheumatology, University of Geneva Hospitals, 4 Rue Gabrielle Perret-Gentil, 1211 Geneva 14, Switzerland

e-mail: stephane.genevay@hcuge.ch

\section{Marty}

Rheumatology Service, Centre Hospitalier Universitaire Henri Mondor, Créteil, France

D. S. Courvoisier

Division of Clinical Epidemiology, Department of Health and Community Medicine, University of Geneva Hospitals, Geneva, Switzerland

V. Foltz $\cdot$ S. Rozenberg

Rheumatology Service, Centre Hospitalier Universitaire

Pitié-Salpétrière, Paris, France

G. Mahieu

Unité du Dos, Centre Hospitalier de Dinant, Dinant, Belgium
Responsiveness and sensitivity to change were assessed through minimal detectable change (MDC), effect size, and Minimal Clinically Important Improvement (MCII). We used an anchor-based method with receiver operating characteristic (ROC) curve analysis to assess MCII and the Patient Acceptable Symptom State.

Results Construct validity, reliability (Cronbach's alpha $=0.87$ ), reproducibility and the absence of floor and ceiling effects were confirmed. Factor analysis indicated a one-dimensional construct that validates the use of a sum score. The MDC (2.1) was inferior to the MCII (2.3). The limit below which the patient claims to be in a fair condition (Patient Acceptable Symptom State) was set at 3. Conclusions The COMI is a self-report questionnaire with the capacity to easily and quickly explore several dimensions in patients with LBP that can be then summarized in a meaningful sum score. Additional knowledge

\section{Demoulin \\ Département des Sciences de la Motricité, Université de Liège, \\ Liège, Belgium}

A. G. Fontana

Clinique de Médecine Physique et Réadaptation, Centre

Hospitalier Universitaire Brugmann, Brussels, Belgium

M. Norberg · P. de Goumoëns

Appareil Locomoteur, Centre Hospitalier Universitaire Vaudois,

Lausanne, Switzerland

C. Cedraschi

Médecine Interne de Réhabilitation, University of Geneva

Hospitals, Geneva, Switzerland 
provided by our study should encourage the widespread use of the COMI among the spine community.

Keywords Low back pain - Multidimensional assessment · Psychometrics · Self-report questionnaire

\section{Introduction}

There are many questionnaires available to assess patients with low back pain (LBP) [1]. Dimensions that are commonly considered important to assess include pain, symptom-specific function, generic well-being, social and work disability, and satisfaction with treatment [2], but using a specific questionnaire for each dimension leads to lengthy assessment that is difficult to achieve in practice. The Core Outcome Measures Index (COMI) was proposed originally to shorten the evaluation time when assessing pain, function, symptom-specific well-being, quality of life, and disability. This 7-item, short and easy to use questionnaire [2] appears to be a reliable and valid [3-5] instrument to assess these five dimensions in LBP patients and it is now routinely used by spine surgeons in the Spine Tango registry (European Spine registry) [6]. The French version of the COMI has been recently validated in patients mainly located in the French-speaking region of Switzerland [4]. However, important psychometric properties (e.g., sensitivity to change) could not be studied in the absence of follow-up after treatment.

The primary aim of this study was to acquire a deeper general knowledge of the measurement characteristics of this questionnaire, e.g., by defining the Patient Acceptable Symptom State (PASS) [7] with a special emphasis on nonsurgical patients. In contrast with our previous study and in order to increase the generalizibility of the results, this study was performed in three different French-speaking populations.

\section{Materials and methods}

\section{Study design/setting}

A prospective 6-month multicenter cohort study was conducted in France, the French-speaking region of Belgium, and the French-speaking region of Switzerland. Patients were recruited from non-surgical spine centers. Inclusion criteria were LBP with or without leg pain for at least 4 weeks, a pain intensity score of at least 3 on a visual analog pain scale ranging from 0 to 10 , and fluency in the French language. Exclusion criteria were a diagnosis of specific LBP (tumor, infection, spondyloarthropathy, or trauma) or the presence of co-morbidities severe enough to interfere with the evaluation of function (e.g., decompensated heart failure, symptomatic knee osteoarthritis). After written informed consent was obtained, patients were asked to complete a questionnaire booklet. To investigate the reproducibility, patients received a shorter booklet with the instruction to complete it a week later at home and then to returned it by mail (short-term follow-up). A full follow-up evaluation was scheduled 4-6 months later. The choice of treatment was left to the decision of each investigator. The sample size was determined according to quality criteria for health status questionnaires [8]. The study was approved by the Institutional Ethics Committee of the University of Geneva Hospitals, Geneva, Switzerland.

\section{Patient-based outcome measures}

The domains included in the COMI are pain symptoms (two items related to back and leg pain, respectively), function, symptom-specific well-being, generic quality of life (QoL) (all in the past week), and work and social disability in the previous month. Pain scores are indicated on a $0-10$ numeric rating scale. Response categories for other items are 5-point adjectival or Likert scales (The French version is available online as a supplementary file; for English version see [9]). The two disability items asked patients to record the number of days that back pain affected their work and daily activities during the previous 4 weeks. These two variables were recorded into categorical variables of five points $(0,1-7,8-14,15-21$, $\geq 22$ days). The pain score is recorded as the higher of the two pain scale scores (back or leg). For the remaining items, each incremental step is allocated 2.5 points and range from 0 ("excellent condition") to 10 ("worst condition"). Scores for social and work disability are averaged to form one disability score. The COMI sum score is computed by the addition of the five subscales (pain, function, symptom-specific well-being, general QoL, and disability) divided by five and thus ranges from 0 ("best health status") to 10 ("worst health status") [5].

In the present study, the French version of the COMI used was identical in the three countries. The validation process of the English to French translation has been previously reported and basic psychometric properties of this version (construct validity and reproducibility) were shown to be acceptable in a small cohort of LBP patients recruited from orthopedic and non-surgical spine centers [4].

At baseline, the questionnaire booklets included questions about sociodemographic variables (age, gender, family status, education, work status), pain characteristics (time since the first episode of LBP, duration of the present episode, previous back surgery, intensity of back-related pain during the past week ranked on a 5-item Likert scale ["no pain" to "extreme pain"]), back pain-related 
disability [French version [10] of the Roland and Morris disability questionnaire (RMDQ)], daily life activity, work and leisure, anxiety and depression, social interest [French version [11] of the Dallas Pain Questionnaire (DPQ), which enables to calculate a subscore for each four subscales as well as the sum score], and health-related QoL [French version [12] of the Euroquol 5 Dimensions Questionnaire (EQ-5D)]. In addition to the COMI, the clinical evolution was evaluated at short-term follow-up by a transition question on a 7-point Likert scale (from "strong improvement" to "strong worsening").

At 6-month follow-up, patients were asked to complete the same questionnaire booklet as at baseline; treatments administered since study inclusion were also recorded. Treatment efficacy was assessed on a 5-point Likert scale (from "no effect" to "excellent effect, almost no symptoms at all") and patient global perceived effect by the same 7-point Likert scale as used at short-term follow-up (from "strong improvement" to "strong deterioration") [13]. Patients were asked also whether they considered their present state as satisfactory through the following question: "Taking into account all what you have to do in your daily life, your pain, and your disability, is your present state satisfactory?" (yes/no answer) [14].

\section{Statistical analysis}

According to recommendations [8], a minimum study sample size of 150 patients was required to ensure sufficient power. Missing data were treated according to the specific recommendations for each questionnaire. COMI scores were computed only when all data were present. Floor and ceiling effects were determined for the COMI total score and for each of the five subscales by computing the percentage of answers at both extremities of the total score and each subscale.

The construct validity of the COMI was explored by investigating the correlations between the COMI subscales and their corresponding validated full-length questionnaire (e.g., RMDQ for the function subscale) using Spearman rank correlation coefficients, corrected for ties. Spearman's Rho coefficients were interpreted as follows: Rho $0.81-1.0=$ "excellent"; $0.61-0.80=$ "very good"; $0.41-0.60=$ "good"; $\quad 0.21-0.40=$ "fair"; and $0-0.20=$ "poor" $[15,16]$. Pre-specified hypotheses were made and good correlations were expected at least between the COMI pain and the 5-item Likert pain scale, the COMI function and the RMDQ or daily life activity subscale of DPQ, the COMI disability and the DPQ work and leisure subscale, the COMI QoL and the EQ-5D, as well as between the COMI sum score and DPQ total score. No specific correlation was expected for the COMI well-being as it has been reported in several studies that this specific scale is not related to other commonly used questionnaires [3-5]. The unidimensionality of the COMI score was first assessed using principal component analysis (PCA). Reliability of the scale was then determined using Cronbach's alpha.

Reproducibility was determined by comparing baseline scores to those reported at short-term follow-up (scheduled 1 week later) among patients who reported no or only minimal change from the time of inclusion. The weighted kappa for single items and the intraclass coefficient of correlation (ICC) for the total score were used, as well as the Bland-Altman plotting method which indicates the smallest detectable difference (SDD; i.e., the amount of detectable change above the random measurement error). The $95 \%$ limits of agreements were calculated by the Bland and Altman method [17] i.e., the mean of the difference between the two measures $\pm 1.96 \times$ the standard deviation (SD) of this difference.

Assessment of the minimal detectable change (MDC) was done by multiplying 1.96 to the difference in score between baseline and short-term follow-up among patients declaring no or minimal improvement $[15,18]$. The minimal clinically important improvement (MCII) was determined using an anchor method based on the patient's assessment in response to the treatment at 6 months by a 5-point Likert scale $(0=$ "no effect", $1=$ "slight effect", $2=$ "moderate effect, could be better, 3 = good effect, still with some symptoms, 4 = excellent effect") [19]. These results were then divided into patients for whom the treatment did not result in any change ( 0 and 1$)$ and those for whom the treatment provided change (2-4). The threshold was determined by subtracting the mean change score of the group of patients who observed a treatment effect from that of the group who did not report any treatment effect. The relationship between the change in COMI sum score and MCII was assessed also by receiver operating characteristic (ROC) curve analysis and the determination of the area under the curve (AUC). The standardized variation of the items and the total score was assessed by effect sizes (mean difference divided by the SD).

PASS was determined using an anchor method based on the patient's answer to the statement: "Taking into account all activities you have to perform in your daily life, your amount of pain, and the level of physical disability, if you were to remain the same for the next months, would this be acceptable for you?" [20]. The threshold for PASS was determined as being the 75th percentile of the COMI sum score at 6-month follow-up of patients answering "yes" to this statement [20]. The relationship between the change in COMI sum score and PASS was also assessed by ROC and AUC curve analyses. 


\section{Results}

Eleven centers recruited 168 patients from May 2009 to June 2010. There were at least two centers in each country (France, Belgium, and Switzerland) recruiting more than 15 patients. The short-term questionnaire (for the reproducibility study) was completed by 138 of 168 patients (mean number of days between baseline and short-term questionnaire, 12.8; SD, 32.0). Long-term follow-up was completed by 142 patients (mean number of months between baseline and long-term follow-up, 5.5; SD 1.5).

Patient baseline characteristics

Patients $(n=168)$ had a mean (SD) age of 45.5 (12.2) years; $56.1 \%$ were female. The current episode of back pain of most patients (82\%) had lasted for more than 3 months (Table 1). Fifteen percent had symptoms and signs compatible with lumbar radiculopathy. Twenty-five patients had undergone previous back surgery (a discectomy for half of them). Pain, function, and QoL-related characteristics of patients at baseline and after treatment at 6-month follow-up are given in Table 2.

Acceptability and floor and ceiling effect

The number of missing items ranges from 2.4 to $3.6 \%$ with $4.8 \%$ of questionnaires having at least one missing item (Table 3). Although several items presented with some significant floor or ceiling effect, no such effect was observed for the COMI sum score (Table 3).

Internal validity

The first PCA of the five items explains $64.3 \%$ of variance. While the eigen value for the second factor was
Table 1 Baseline characteristics of patients $(n=168)$

$L B P$ low back pain

a According to the Paris Task Force classification [26]

\begin{tabular}{|c|c|c|}
\hline Characteristics & Categories & $N(\%)$ \\
\hline Gender, female $(n=168)$ & & $96(56.1)$ \\
\hline \multirow[t]{4}{*}{ Type of $\operatorname{LBP}^{\mathrm{a}}(n=158)$} & LBP without radiating pain & $76(48.1)$ \\
\hline & Non-specific radiation below gluteal fold & $31(19.6)$ \\
\hline & Non-specific radiation below the knee & $27(17.1)$ \\
\hline & Radicular pain & $24(15.2)$ \\
\hline \multirow[t]{5}{*}{ Duration of pain $(n=164)$} & 4-7 weeks & $18(11.0)$ \\
\hline & 7 weeks -3 months & $11(6.7)$ \\
\hline & $3-6$ months & $30(18.3)$ \\
\hline & $6-18$ months & $32(19.5)$ \\
\hline & $>18$ months & $73(44.5)$ \\
\hline Previous episode of LBP $(n=168)$ & & $138(85.2)$ \\
\hline \multirow{3}{*}{ Level of education $(n=160)$} & Obligatory schooling ( 9 years of education) & $36(22.5)$ \\
\hline & Professional diploma & $58(36.3)$ \\
\hline & University & $66(41.3)$ \\
\hline \multirow{3}{*}{ Type of usual work $(n=159)$} & Sedentary & $48(30.2)$ \\
\hline & Physical & $61(38.4)$ \\
\hline & A mix of both & $50(31.4)$ \\
\hline \multirow{6}{*}{ Work status $(n=163)$} & Employed & $90(55.2)$ \\
\hline & Unemployed & $11(6.7)$ \\
\hline & Insurance beneficiary (disease, accident, invalidity) & $42(25.8)$ \\
\hline & Retired & $10(6.1)$ \\
\hline & No paid activity & $5(3.1)$ \\
\hline & Other & $5(3.1)$ \\
\hline Duration of sick leave & None & $51(32.9)$ \\
\hline \multirow[t]{6}{*}{$(n=155)$} & $<7$ weeks & $24(15.5)$ \\
\hline & 7 weeks -3 months & $10(6.5)$ \\
\hline & $3-6$ months & $19(12.3)$ \\
\hline & $6-18$ months & $19(12.3)$ \\
\hline & $>18$ months & $16(10.3)$ \\
\hline & Not applicable & $16(10.3)$ \\
\hline
\end{tabular}


slightly above 1 , the screen plot clearly favored a onefactor solution. Reliability measured by Cronbach's alpha was 0.87 .

\section{Construct validity}

All hypotheses were fulfilled for construct validity. The COMI sum score and all subscales of the COMI, except COMI well-being, had a good or very good correlation with their respective reference questionnaire ranging from 0.52 (between COMI function and RMDQ) to 0.65 (between COMI sum score and DPQ sum score). As expected, COMI well-being showed a low correlation with all reference questionnaires, the highest correlation being with EQ-5D (0.39).

Table 2 Pain, function, and quality of life-related characteristics of patients at baseline and after treatment at 6-month follow-up (mean [SD])

\begin{tabular}{lcc}
\hline & $\begin{array}{l}\text { Baseline } \\
(n=168)\end{array}$ & $\begin{array}{c}\text { Follow-up } \\
(n=142)\end{array}$ \\
\hline Back pain (0-10) & $5.5(2.0)$ & $3.7(2.6)$ \\
Leg pain (0-10) & $3.6(2.9)$ & $2.6(2.8)$ \\
Roland and Morris disability & $12.9(5.0)$ & $7.5(6.5)$ \\
$\quad$ questionnaire (0-24) & & \\
Dallas pain questionnaire & & \\
Daily activities (0-100) & $60.5(17.6)$ & $40.6(25.9)$ \\
Work and leisure (0-100) & $57.8(23.5)$ & $37.2(29.8)$ \\
Anxiety and depression (0-100) & $42.5(26.4)$ & $29.4(28.6)$ \\
Social interest (0-100) & $34.1(24.2)$ & $24.5(24.7)$ \\
Euroquol 5 dimensions questionnaire & $0.4(0.2)$ & $0.6(0.3)$ \\
$\quad(0-1)$ & & \\
COMI sum score & $6.3(1.8)$ & $4.0(2.6)$ \\
\hline
\end{tabular}

Reproducibility

Of the 138 patients who responded to the short-term follow-up questionnaire, 132 reported no or only minimal change from inclusion and were thus included in the testretest analysis. Test-retest agreement was high for all items (range 0.66-0.88) except for the item on well-being (weighted kappa, 0.48). The test-retest agreement for the total score was very high at 0.81 (95\% CI, 0.74-0.86). The Bland-Altman plotting method indicating the SDD was 2.09 (Fig. 1).

Responsiveness, sensitivity to change, and additional characteristics

The MDC for single items was less than 2.5 points on the 10-point scale, except for the questions on pain (MDC for COMI back pain $=2.9$; COMI leg pain = 3.7; COMI pain = 2.7). The MDC for the COMI sum score in this population was 2.1. However, the mean difference between scores among stable patients was very low. By contrast, the MCII for the sum score was 2.3. The AUC for the prediction of patient's own assessment in response to treatment by the change in COMI sum score was 0.80 , meaning that a patient reporting no or a slight effect had a $80 \%$ chance of having a lower COMI sum score change than a patient who reported at least a moderate treatment effect. The effect size of the COMI sum score was 1.01.

The PASS for the sum score (scale from 0 to 10) was 3.05. This threshold on the COMI sum score at follow-up correctly classified $90.6 \%$ of the patients who declared to be dissatisfied with their present state and $74.3 \%$ of patients reporting as satisfied. The AUC for the prediction of PASS by the COMI sum score at follow-up was 0.84 (Fig. 2), meaning that a patient who is dissatisfied with his/
Table 3 Item characteristics of the Core Outcome Measures Index (COMI) at baseline $(n=168$ patients)

$S D$ standard deviation, $P C A$ principal component analysis

${ }^{a}$ Each scale rated from 0 to 10

\begin{tabular}{|c|c|c|c|c|c|c|c|}
\hline & $\begin{array}{l}\text { Missing } \\
(\%)\end{array}$ & $\begin{array}{l}\% \text { at lowest } \\
\text { value }\end{array}$ & $\begin{array}{l}\% \text { at highest } \\
\text { value }\end{array}$ & $\begin{array}{l}\text { Lowest } \\
\text { value }\end{array}$ & $\begin{array}{l}\text { Highest } \\
\text { value }\end{array}$ & $\begin{array}{l}\text { Mean }^{a} \\
(\mathrm{SD})\end{array}$ & $\begin{array}{l}\text { Loading } \\
\text { of PCA }\end{array}$ \\
\hline COMI back pain & 3.0 & 0.6 & 0.6 & 0 & 10 & $5.5(2.0)$ & - \\
\hline COMI leg pain & 3.6 & 21.4 & 0.6 & 0 & 10 & $3.6(2.9)$ & - \\
\hline COMI pain & 2.4 & 0.0 & 1.2 & 2 & 10 & $6.0(1.9)$ & 0.70 \\
\hline $\begin{array}{l}\text { COMI social } \\
\text { disability }\end{array}$ & 3.0 & 13.7 & 32.1 & 0 & 10 & $5.6(3.7)$ & - \\
\hline COMI work disability & 3.0 & 33.9 & 31.0 & 0 & 10 & $4.5(4.3)$ & - \\
\hline COMI disability & 3.0 & 13.1 & 25.0 & 0 & 10 & $5.1(3.7)$ & 0.60 \\
\hline COMI function & 2.4 & 0.6 & 9.5 & 0 & 10 & $6.0(2.1)$ & 0.87 \\
\hline COMI well-being & 2.4 & 0.0 & 49.4 & 2.5 & 10 & $8.4(1.9)$ & 0.59 \\
\hline COMI quality of life & 2.4 & 1.2 & 7.7 & 0 & 10 & $5.8(2.1)$ & 0.78 \\
\hline COMI sum score & 4.8 & 0.0 & 0.6 & 2.4 & 10 & $6.3(1.8)$ & - \\
\hline
\end{tabular}


Fig. 1 Bland-Altman plotting showing limits of agreement between the Core Outcome Measures Index (COMI) mean score at baseline and at short term (average time 12 days) among stable patients

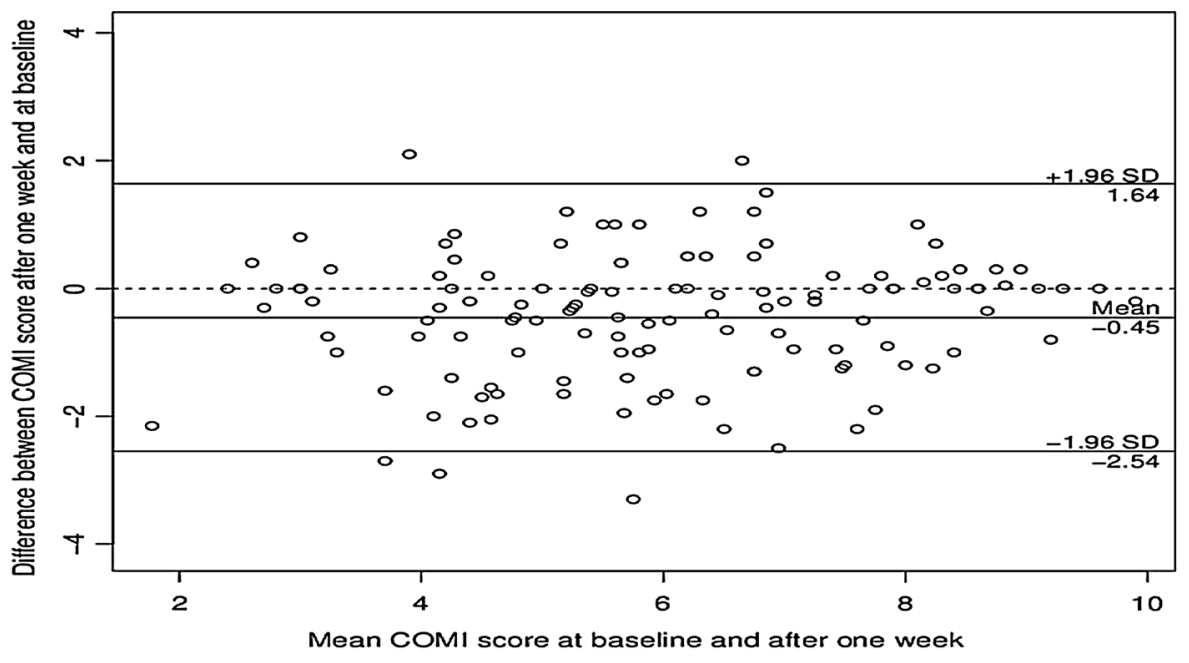

cohort of non-surgical patients. The recruitment performed in three different French-speaking countries is an important point for the generalizability of the questionnaire. More importantly, it provides additional clinically meaningful psychometric properties that have not been previously reported in any other language [3-5]. It is the first time that a factor analysis is reported for the COMI. Although it is in essence a multidimensional tool, this analysis surprisingly indicates that the COMI has a one-dimensional construct. We hypothesize that this may refer to the fact that this questionnaire captures something unique for all these patients (i.e., they are all suffering from LBP) and might indicate that the investigated dimensions have been adequately chosen to provide a comprehensive evaluation of these patients. Importantly, this finding validates the use of a sum score that effectively represents the patient's global state. Interestingly, this has been already reported for a version of the COMI specifically developed for neck pain patients [21].

PASS is an emerging concept that has recently been reported for other self-report questionnaires in the field of musculoskeletal diseases [22, 23], but was not previously determined for the COMI. Patients with a COMI sum score equal or inferior to 3 can be confidently considered as having reached an acceptable symptom state $(\mathrm{AUC}=0.84)$. Complementary to MCII, which characterizes an improvement from a previous state, PASS is characteristic of a present state of being. PASS appears to be less influenced by baseline characteristics than MCII [23] and to be stable over time [24]. PASS scores are increasingly used to report results in clinical trials [25] and are proposed as a tool to help guide clinical and surgical decisions [26].

In our patient cohort, the value from which an improvement can be considered to have clinical relevance (MCII) is 2.3. The fact that the MCII is above the MDC 
(2.1) confirms that MCII can be adequately used in clinical research and practice. The AUC (0.80) for the change in the COMI sum score is high and shows a good ability to predict the patient's assessment in response to the treatment at 6 months. The value of MCII in this study is similar to the value found (2.6) in a large cohort of surgical patients [27]. The effect size of the COMI sum score (1.01) is large and similar to that obtained in other studies [3, 5], thus indicating that this questionnaire has also a good responsiveness (sensitivity to change) in non-surgical patients.

Some of the new psychometric properties of the COMI described in this study, like the PASS score, should be replicated in other populations (e.g., surgical patients) before being generalized. Other important results like factor analysis should not be influenced by translation or clinical characteristics of the patients and are thus be valid for all translations. Lastly, for parameters like ICC MDC and MCII, our results confirm those reported in other crosscultural adaptations and thus can be considered reliable [3, 5, 9, 28, 29].

In conclusion, the French cultural adaptation of this selfreport questionnaire has adequate psychometric properties to study and follow-up subacute and chronic LBP patients in large cohort studies as presently done in Spine Tango [6]. Furthermore, considering its intrinsic qualities, namely brevity, multidimensionality, ease of application and scoring, the COMI has all the necessary criteria to be used in daily practice care by spine specialists or even general practitioners. The COMI is a useful instrument providing a comprehensive evaluation that helps to document treatment efficacy in a patient population notoriously difficult to treat.

Acknowledgments We thank all the team from "Nukleus" and, in particular, Mrs V. Gordin and M. Demonnet for their help and logistic support. We wish also to thank members of the Spine section of the French Rheumatology Society for their support in recruiting patients and Pfizer AG for their financial support. This study was supported by an unrestricted educational grant from Pfizer.

Conflict of interest None.

\section{References}

1. Cleland JJ, Gillani RR, Bienen EJR, Sadosky AA (2011) Assessing dimensionality and responsiveness of outcomes measures for patients with low back pain. Pain pract 11(1):57-69

2. Deyo RA, Battie M, Beurskens AJ, Bombardier C, Croft P, Koes B, Malmivaara A, Roland M, Von Korff M, Waddell G (1998) Outcome measures for low back pain research. A proposal for standardized use. Spine 23(18):2003-2013

3. Ferrer M, Pellise F, Escudero O, Alvarez L, Pont A, Alonso J, Deyo R (2006) Validation of a minimum outcome core set in the evaluation of patients with back pain. Spine 31(12):1372-1379 discussion 1380

4. Genevay S, Cedraschi C, Marty M, Rozenberg S, De-Goumons P, Faundez A, Balagu F, Porchet F, Mannion AFA (2012) Reliability and validity of the cross-culturally adapted French version of the core outcome measures index (COMI) in patients with low back pain. Eur Spine J 21(1):130-137

5. Mannion AF, Elfering A, Staerkle R, Junge A, Grob D, Semmer NK, Jacobshagen N, Dvorak J, Boos N (2005) Outcome assessment in low back pain: how low can you go? Eur Spine J 14(10):1014-1026

6. Roeder C, Chavanne A, Mannion AF, Grob D, Aebi M (2005) SSE Spine Tango-content, workflow, set-up. www.eurospine.org-Spine Tango. Eur Spine J 14(10):920-924

7. Kvien TK, Heiberg T, Hagen KB (2007) Minimal clinically important improvement/difference (MCII/MCID) and patient acceptable symptom state (PASS): what do these concepts mean? Ann Rheum Dis 66(Suppl 3):iii40-iii41

8. Terwee CB, Bot SD, de Boer MR, van der Windt DA, Knol DL, Dekker J, Bouter LM, de Vet HC (2007) Quality criteria were proposed for measurement properties of health status questionnaires. J Clin Epidemiol 60(1):34-42

9. Storheim K, Brox JI, Lochting I, Werner EL, Grotle M (2012) Cross-cultural adaptation and validation of the Norwegian version of the Core Outcome Measures Index for low back pain. Eur Spine J 21(12):2539-2549

10. Coste J, Le Parc JM, Berge E, Delecoeuillerie G, Paolaggi JB (1993) French validation of a disability rating scale for the evaluation of low back pain (EIFEL questionnaire). Rev Rhum Ed Fr 60(5):335-341

11. Marty M, Blotman F, Avouac B, Rozenberg S, Valat JP (1998) Validation of the French version of the Dallas Pain Questionnaire in chronic low back pain patients. Rev Rhum Engl Ed 65(2):126-134

12. Perneger TV, Combescure C, Courvoisier DS (2010) General population reference values for the French version of the EuroQol EQ-5D health utility instrument. Value Health 13(5):631-635

13. Beurskens AJ, de Vet HC, Koke AJ (1996) Responsiveness of functional status in low back pain: a comparison of different instruments. Pain 65(1):71-76

14. Tubach F, Ravaud P, Beaton D, Boers M, Bombardier C, Felson DT, van der Heijde D, Wells G, Dougados M (2007) Minimal clinically important improvement and patient acceptable symptom state for subjective outcome measures in rheumatic disorders. J Rheumatol 34(5):1188-1193

15. Cohen J (1988) Statistical power analysis for the behavioral sciences, 2nd edn. Lawrence Erlbaum Associates, Hillsdale

16. Steiner D, Norman G (1995) Health measurement scales: a practical guide to their development and use. Oxford Medical Publications, Oxford

17. Bland JM, Altman DG (1986) Statistical methods for assessing agreement between two methods of clinical measurement. Lancet 1(8476):307-310

18. Hopkins WG (2000) Measures of reliability in sports medicine and science. Sports med 30(1):1-15

19. Tubach F, Ravaud P, Baron G, Falissard B, Logeart I, Bellamy N, Bombardier C, Felson D, Hochberg $M$, van der Heijde D, Dougados M (2005) Evaluation of clinically relevant changes in patient reported outcomes in knee and hip osteoarthritis: the minimal clinically important improvement. Ann Rheum Dis 64(1):29-33

20. Tubach F, Wells GA, Ravaud P, Dougados M (2005) Minimal clinically important difference, low disease activity state, and patient acceptable symptom state: methodological issues. J Rheumatol 32(10):2025-2029

21. Qiao J, Zhu F, Zhu Z, Xu L, Wang B, Yu Y, Qian BP, Ding Y, Qiu Y (2013) Validation of the simplified Chinese version of the 
Core Outcome Measures Index (COMI). Eur Spine J 22(12):2821-2826

22. Escobar A, Gonzalez M, Quintana JM, Vrotsou K, Bilbao A, Herrera-Espineira C, Garcia-Perez L, Aizpuru F, Sarasqueta C (2012) Patient acceptable symptom state and OMERACT-OARSI set of responder criteria in joint replacement. Identification of cutoff values. Osteoarthr Cartil 20(2):87-92

23. Tubach F, Dougados M, Falissard B, Baron G, Logeart I, Ravaud P (2006) Feeling good rather than feeling better matters more to patients. Arthritis Rheum 55(4):526-530

24. Tubach F, Pham T, Skomsvoll JF, Mikkelsen K, Bjorneboe O, Ravaud P, Dougados M, Kvien TK (2006) Stability of the patient acceptable symptomatic state over time in outcome criteria in ankylosing spondylitis. Arthritis Rheum 55(6):960-963

25. Hochberg MCM, Wohlreich MM, Gaynor PP, Hanna SS, Risser RR (2011) Clinically relevant outcomes based on analysis of pooled aata from 2 trials of Duloxetine in patients with knee Osteoarthritis. J Rheumatol 39(2):352-358

26. Quintana JMJ, Aguirre UU, Barrio II, Orive MM, Garcia SS, Escobar AA (2011) Outcomes after total hip replacement based on patients' basal status, what results you can expect. Arthritis Care Res (Hoboken) 64(4):563-572

27. Mannion AF, Porchet F, Kleinstuck FS, Lattig F, Jeszenszky D, Bartanusz V, Dvorak J, Grob D (2009) The quality of spine surgery from the patient's perspective: part 2. Minimal clinically important difference for improvement and deterioration as measured with the Core Outcome Measures Index. Eur Spine J 18(Suppl 3):374-379

28. Mannion AF, Boneschi M, Teli M, Luca A, Zaina F, Negrini S, Schulz PJ (2012) Reliability and validity of the cross-culturally adapted Italian version of the Core Outcome Measures Index. Eur Spine J 21(Suppl 6):S737-S749

29. Miekisiak G, Banach M, Kiwic G, Kubaszewski L, Kaczmarczyk J, Sulewski A, Kloc W, Libionka W, Latka D, Kollataj M, Zaluski R (2014) Reliability and validity of the Polish version of the Core Outcome Measures Index for the neck. Eur Spine J 23(4):898-903 